\title{
BMJ Open Improving skills and care standards in the support workforce for older people: a realist review
}

\author{
Jo Rycroft-Malone, ${ }^{1}$ Christopher Burton, ${ }^{1}$ Beth Hall, ${ }^{2}$ Brendan McCormack, ${ }^{3}$ \\ Sandra Nutley, ${ }^{4}$ Diane Seddon, ${ }^{5}$ Lynne Williams ${ }^{1}$
}

To cite: Rycroft-Malone J, Burton C, Hall B, et al. Improving skills and care standards in the support workforce for older people: a realist review. BMJ Open 2014;4:e005356.

doi:10.1136/bmjopen-2014005356

- Prepublication history for this paper is available online. To view these files please visit the journal online (http://dx.doi.org/10.1136/ bmjopen-2014-005356).

Received 28 March 2014 Revised 8 May 2014 Accepted 9 May 2014

\section{CrossMark}

\begin{abstract}
${ }^{1}$ School of Healthcare Sciences, Bangor University, Bangor, UK

${ }^{2}$ College of Physical and Applied Sciences, Bangor University, Bangor, UK

${ }^{3}$ School of Health Sciences, Queen Margaret University, Edinburgh, UK

${ }^{4}$ School of Management, University of St Andrews, St Andrews, UK

${ }^{5}$ School of Social Sciences, Bangor University, Bangor, UK
\end{abstract}

\section{Correspondence to}

Professor Jo Rycroft-Malone; j.rycroft-malone@bangor.ac.uk

\section{ABSTRACT}

Introduction: In the context of a population that is growing older, and a number of high-profile scandals about care standards in hospital and community settings, having a skilled and knowledgeable workforce caring for older people is an ethical and policy imperative. Support workers make up the majority of the workforce in health and social care services for older people (aged 65 years and over), and yet little is known about the best way to facilitate their development. Given this gap, this review will draw on evidence to address the question: how can workforce development interventions improve the skills and the care standards of support workers within older people's health and social care services?

Methods and analysis: As we are interested in how and why workforce development interventions might work, in what circumstances and with whom, we will conduct a realist review, sourcing evidence from health, social care, policing and education. The review will be conducted in four steps over 18 months to (1) construct a theoretical framework, that is, the review's programme theories; (2) retrieve, review and synthesise evidence relating to interventions designed to develop the support workforce guided by the programme theories; (3) 'test out' our synthesis findings and refine the programme theories, establish their practical relevance/potential for implementation and (4) formulate recommendations about improvements to current workforce development interventions to contribute to the improvement of care standards in older people's health and social care services, potentially transferrable to other services.

Ethics and dissemination: Ethical approval is not required to undertake this review. Knowledge exchange activities through stakeholder engagement and online postings are embedded throughout the lifetime of the project. The main output from this review will be a new theory driven framework for skill development for the support workforce in health and social care for older people.

Trial registration number: CRD42013006283.

\section{INTRODUCTION}

The UK population is getting older-by 2031 it is estimated that one in five people will be

\section{Strengths and limitations of this study}

Realist review that will inform a broad range of stakeholders including health and social care policy makers, managers and the public about how the skills and knowledge of the support workforce can be enhanced to improve the quality of older people's services.

- Argues for the appropriateness and fit of the realist review approach to evaluate complex interventions in the development of the health and social care support workforce.

- There is a pressing need for research that identifies interventions that can improve the skills and knowledge of the support workforce in order to promote safe, effective and responsive personcentred care to older people.

over 65 years old. ${ }^{1}$ Older people are the main recipients of care in the National Health Service (NHS), and older people's care costs the UK NHS relatively more than those of working age. ${ }^{2}$ Research suggests that older people require care which encompasses health and social care functions. ${ }^{3}$ Multiple, long-term conditions experienced by older people may be associated with a complex mix of interventions and approaches, including specific needs around communication and cognition, which will shape the design of hospital-based and community-based care interventions. The rapid increase in the older person population is driving the current pressures to develop new service models, processes, roles and expertise for delivering effective and efficient care for this group, where people have distinctive, often individualised care needs. As part of this, greater use and development of the support workforce in health and social care is likely to remain a long-term priority for NHS managers and other sector organisations.

High-quality care provision for older people is a strategic priority, and points to 
the need for appropriate workforce development interventions to nurture and support the development of person-centred care across health and social care settings. A recent series of investigations and high-profile cases have questioned current practices in services provided to older people. These include a Care Quality Commission report ${ }^{4}$ which identified concerns over the skills, training and availability of the care workforce within hospital settings to deliver dignified and appropriate care. This followed on from several other critical reports of the standards of care offered to older patients within the NHS, including a particularly shocking investigation by the Parliamentary and Health Service Ombudsman, ${ }^{5}$ which has called for standards of NHS care for older people to be improved, and others which have accused the NHS of 'ageist' practices and attitudes. ${ }^{6}{ }^{7}$ Likewise, the preferences and experiences of older people may not always be reflected in care policies, structures and practices. 89

The health and social care support workforce is defined as providers of 'face-to-face care or support of a personal or confidential nature to service users in clinical or therapeutic settings, community facilities or domiciliary settings, but who do not hold qualifications accredited by a professional association and are not formally regulated by a statutory body'. ${ }^{10}$ The support workforce delivers care alongside the regulated, professional workforce in their day-to-day duties. However, their use and role development has been somewhat ad $\operatorname{hoc}^{11}$ and largely dependent on the various activities they perform. ${ }^{12}$ In parallel, support workers have also become an undervalued resource. ${ }^{13}$ Additionally, there is a lack of clarity about the role of support workers, with their roles developing organically rather than systematically and consequently their preparation and continuing development tend to be haphazard. ${ }^{14}$

There is no unified body of evidence to indicate how to enhance workforce development interventions for improving the skills and care standards in the support workforce. Workforce development in this context includes the support required to equip those providing care to older people with the right skills, knowledge and behaviours to deliver safe and high-quality services. ${ }^{15}$ Evidence about interventions to develop the health and social care support workforce for older people is limited, and further research is urgently needed to inform service about how to improve standards for the future. ${ }^{14}$ In part, this reflects the lack of a common definition of the support workers' role, largely due to the variety of duties that they perform, ${ }^{12}$ and the different approaches to workforce design and development models that NHS trusts and other services have adopted. ${ }^{11}$ This diversity and lack of clarity means that often support workers are 'figuring it out in the moment', delivering care that may not be appropriate or evidence informed. ${ }^{16}$

This review will address a gap in the evidence base by identifying interventions at individual, team and organisational levels that have the potential to enhance the skills and care standards in the support workforce for older people. While a small number of scoping reviews in health and social care have focused on aspects of, for example, support workers' roles, tasks and regulation, ${ }^{17} 18$ we are not aware of systematic reviews of the effectiveness of support workforce development interventions. For this review, adopting a realist approach to the systematic reviewing of evidence will uncover how and why workforce development interventions may impact, and on whom; to guide future workforce development policy and practice.

\section{BACKGROUND}

Within health and social care, the support workforce is large; an estimated 1.3 million working on the frontline of care $^{19}$ and can be categorised under the different types of role they perform, including direct care, indirect care, administration and facilitation. ${ }^{20} 21$ While growth in the support workforce has sometimes been driven by initiatives to reduce costs, which has involved role substitution for regulated staff, there is evidence to show that support workers can act as an additional resource to enhance older people's experiences by improving the contact with care practitioners. ${ }^{22}{ }^{23}$ The findings from a number of studies point to the need to improve the skills and training approaches currently used to develop support workers. ${ }^{45}$ There is evidence to suggest that support workers are not used as effectively as possible and are often undervalued. ${ }^{13}{ }^{20}$ Recommendations from the Commission for Dignity in Care $^{7}$ include the need to shift to more work-based approaches to learning and development for all staff, including the support workforce. Research concerning the support workforce has generally focused on their role and contribution in the acute care sector, ${ }^{6}$ patients' care needs in particular situations such as dementia services ${ }^{24}$ or the relationships between support workers and different professional groups. ${ }^{11} 1320$ Only one study has specifically examined support workers in older people's services. ${ }^{23}$

Previous work on the development of professionals has focused on advancing workers from novices to experts. $^{25}$ However, such models of education have focused on individuals who are already highly educated and with additional years of experience to build on, which is often not the case for the support workforce. Additionally, much of this work focuses on how professionals learn, including the different processes for adopting new practices, rather than on considering contextual constraints, such as the role of organisational strategy and professional regulation. The degree of synergy between workforce development strategies and opportunities for job and role development is also uncertain. The general lack of clarity and diversity in models, roles and care settings have resulted in a gap in knowledge about what makes for effective interventions 
for the development of the support workforce. This review will fill this gap by providing actionable and transferable findings from a realist review of evidence relating to the development of the support workforce in different settings (health, social care, policing and education) in order to uncover what workforce development interventions are effective in improving the care received by older people. The review will be of direct benefit to health and social care through providing a resource to inform the development of support workers, and helping to address some of the failures in the quality of services provided to older people identified by previous investigations. ${ }^{4} 67$

\section{REVIEW QUESTION AND AIMS}

Research question: How can workforce development interventions improve skills and care standards of support workers within older people's health and social care services?

The main aims are to:

1. Identify support worker development interventions from different public services and to synthesise evidence of impact.

2. Identify the mechanisms through which these interventions deliver support workforce and organisational improvements that are likely to benefit the care of older people.

3. Investigate the contextual characteristics that will mediate the potential impact of these mechanisms on clinical care standards for older people.

4. Develop an explanatory framework that synthesises review findings of relevance to services delivering care to older people.

5. Recommend improvements for the design and implementation of workforce development interventions for support workers.

Workforce development interventions are characteristic of complex social programmes with inter-related components, the impacts of which are likely to be contingent on multiple personal, work-related and organisational factors. Synthesising evidence of 'what works' in this situation requires an approach that can accommodate this complexity and contingency. A realist review adopts a theory-driven approach to evidence synthesis, underpinned by a realist philosophy of science and causality. ${ }^{26}$ Causal explanations are expressed as contingent relationships between mechanisms (changes in participants' reasoning or resources), context (contingencies) and outcomes, often abbreviated to context-mechanismoutcome configuration (CMO) to show how particular contexts or conditions trigger or fire mechanisms to generate an observed outcome.

The CMO framework can be used in abstract ways to explain broad processes, or in more specific ways to examine how programmes work. ${ }^{27}$ Realist reviews explore complex social programmes and seek out mid-range theories that explain observable patterns of outcomes (demi-regularities), including why interventions are successful in some settings but not in others. ${ }^{28} 29$ Strong stakeholder engagement strategies are used to ensure interpretive depth and the policy relevance of synthesis findings, and require the consideration of a much broader and heterogeneous evidence base than traditional Cochrane reviews of effectiveness. ${ }^{30}{ }^{31}$ Realist review methods have been developing, ${ }^{32}$ including through the work of members of this project team, ${ }^{31} 33$ and are becoming increasingly used in generating explanatory evidence about the workings of complex, contextually contingent programmes and interventions.

\section{THEORETICAL TERRITORY}

The review will establish a mid-range programme theory or theories which will provide an evidence-based account of how workforce development programmes work. The initial programme theories will be developed in the first phase of the review, informed by the commissioning brief, extant literature and theory building work with stakeholders including the support workforce. The review will employ a blended approach to theory construction, so that the development of the programme theory is informed by stakeholders' perspectives in addition to established theories, which will orient explanation building. Our initial work indicates the relevance of a number of relevant, interlinked theoretical disciplines for the development of the programme theories, each with their own literature, approaches and concerns. These include:

- Theories of professional learning and role progression, including the development of expertise ${ }^{2534}$;

- Theories of adult and transformational learning ${ }^{35} 36$;

- Workforce development implementation, including connections between different development interventions and workforce functions;

- Theories of behaviour change, ${ }^{37} 38$ practice development $^{39}$ and knowledge utilisation ${ }^{40}{ }^{41}$;

- The role of organisational and other contextual influences, such as structural factors which affect the implementation of learning and practices. ${ }^{42-44}$

Additionally, we are interested in identifying the different impacts that workforce interventions could potentially have, including to knowledge, attitudes, skills and behaviour. However, we recognise that, for example, an increase in knowledge about an issue may not result in a change of behaviour (ie, better standards of care) but may be a precursor to behaviour change. Therefore, in this review, we will conceptualise impact as a continuum ranging from conceptual to instrumental or direct impact: that is, from awareness, knowledge and understanding, attitudes and perceptions, to practice change. ${ }^{41}$

\section{METHODS}

Reflecting emerging frameworks for reporting realist reviews, ${ }^{26}$ this review will be conducted in four interlinked phases over 18 months: 
1. Programme theory development.

2. Evidence retrieval, data extraction and synthesis.

3. Programme theory testing and refinement through evidence synthesis.

4. Development of actionable recommendations.

Stakeholder engagement is embedded throughout each phase. We will form an advisory group of representatives from organisations associated with the design, commissioning, delivery and experience of workforce development programmes for the support workforce for older people. This group will be complemented by representatives from advocacy organisations representing the health and social care interests of older people. The group will be responsible for advising on the relevance of review questions, interpretation of findings and the dissemination of synthesis findings. We will ensure that mobilisation of the knowledge generated around the focus of the evidence synthesis and the realist review processes adopted is mobilised across the lifetime of the project through the use of social media; formal dissemination activities; policy, practice and workforce engagement events.

\section{Phase 1: Programme theory development}

An initial programme theory will be developed through stakeholder engagement, and an overview of relevant extant theory. We will hold a theory-building workshop with stakeholders including educators, practitioners, managers and service user representatives to identify and prioritise the theory to be evaluated in the review.

\section{Phase 2: Evidence retrieval, data extraction and evidence synthesis}

Our review process will involve searching for evidence relevant to 'testing' and refining the initial programme theory, and extracting data from the sources of evidence identified. Older people access a wide range of generalist and specialist services to address their health and social care needs. Our approach will be to target services specific to older people in the first instance across hospital, community and third sector care providers. In the first instance, we will target evidence relevant to the health and social care support workforce including advocacy organisations (eg, Age UK, The Alzheimer's Society).

We will focus on interventions that address the knowledge and skills required by this workforce to contribute to health and social care for older people in generalist and specialist settings. The realist review provides an ideal approach for testing the robustness of emerging findings from one body of literature to another, and provides the opportunity to see if other literatures offer different learning and mechanisms, which are transferable to the health and social assistant care workforce. Our initial search of the literature in health and social care will be complemented by more purposeful searches for support worker development interventions in the wider public service fields of policing and education. Searches in these other literatures will be targeted to enable us to refine the emerging findings from the health and social care literature.

\section{Search strategy}

One strength of the realist review approach is that the evidence base to be reviewed and synthesised can be broad and eclectic. ${ }^{30}$ In fact, a diversity of evidence provides an opportunity for richer mining and greater explanation. To maximise relevance, our search will be limited to material from 1986 to 2013, which includes the last two major workforce development shifts within the UK health and social care workforce. We intend to include material indexed in the major health, social and welfare databases using keywords identified in previous systematic reviews and database specific 'keywords' adapted for each information source. The range of databases, including grey literature databases are specified in box 1 .

\section{Inclusion and exclusion criteria}

We will include reports of workforce, practice and/or organisational development programmes and interventions (and also in combinations). In contrast to other approaches, in a realist review, evidence is not excluded (unless it does not relate to the programme theory or theories). However, in this review we will not search for or include evidence that may have limited transferability to the NHS such as health systems within low-income countries. The test for inclusion will be the realist one: is the evidence provided 'good and relevant enough' to be included, ${ }^{30}$ to inform the development of CMOs. Discrepancies in opinions about the relevance of articles will be resolved through discussion among the project team.

The search for references will be augmented by searches for support worker role evaluations or intervention

\section{Box 1 Review range of databases}

Table of search databases
MEDLINE
Web of Science
Zetoc
CINAHL
- AMED
HMIC
NHS Evidence
Cochrane
DARE
- HTA
NEED
- Social Care Online
Psyclnfo
- ASSIA
- Social Services Abstracts
- Sociological Abstracts
- Google Scholar
OpenGrey

Table of search databases

- MEDLINE

Web of Science

Zetoc

- CINAHL

- AMED

HMIC

- NHS Evidence

- Cochrane

DARE

HTA

NEED

- Social Care Online

- Psyclnfo

- ASSIA

Social Services Abstracts

- OpenGrey 
research which makes specific reference to how workforce interventions are embedded. We will also conduct internet-based searches for grey literature, such as workforce development project reports; national inspection and regulation quality reports; evaluative information about these initiatives. We will also use snowballing techniques and draw on the expertise of the project advisory group, other key researchers and educators and organisations to ensure we have not missed evidence that might not be visible through traditional methods.

\section{Data extraction}

The programme theories being 'tested' through the review are made visible through the data extraction forms. ${ }^{31}$ A bespoke set of data extraction forms will be developed based on the content of the programme theories, which thereby provides a template to interrogate the programme theories. The data extraction form will also include details about the study-such as approach to data collection and analysis and information about the sample (s). If the evidence meets the test of relevance, data will be extracted using the bespoke pro forma and then checked by a second member of the team.

\section{Synthesis}

The analytical task involves synthesising across the extracted information the relationships between emerging mechanisms, contexts and outcomes. Through our previous experience of realist review, ${ }^{31} 33$ and building on the suggestions of Pawson ${ }^{30}$ and principles of realist enquiry, we have developed an approach to synthesis that includes:

- Organisation of extracted information into evidence tables representing the different bodies of literature (eg, health, teaching, social care, policing);

- Theming across the evidence tables in relation to emerging demi-regularities (patterns) among CMO configurations seeking confirming and disconfirming evidence;

- Linking these demi-regularities to develop hypotheses.

The resultant hypotheses act as synthesised statements of findings around which a mid-range theoretical, contingent narrative can be developed summarising the characteristics of the evidence underpinning workforce development programmes. Outputs from this phase will be a comprehensive evidence base related to workforce development for the support workforce, which we will make publicly available, and a set of mid-range hypotheses supported by relevant evidence which will be further refined in phase 3 .

\section{Phase 3: Testing and refining programme theories}

To enhance the trustworthiness of the resultant programme theories from the evidence review, as well as facilitate the development of a final review narrative, we will conduct up to 10 semistructured audiorecorded telephone interviews with stakeholders, including members of the support workforce. These participants will be purposively sampled to obtain different perspectives relevant to the review question. Interviewees will include service delivery managers, policy makers, education providers, commissioners and support workers. An interview schedule will be developed based on the findings that have emerged from the synthesis process and will aim to elicit stakeholders' views on their resonance.

\section{Phase 4: Actionable recommendations}

We will work with the Project Advisory Group including representation of the support workforce and patient and public participants to develop a set of actionable recommendations and the development of an evidence informed framework of what works for whom and in what context in relation to workforce development interventions for the clinical support workforce for older people. This will be achieved through one face-to-face meeting, and virtual meetings via teleconference. We will also hold a knowledge mobilisation event with a group of stakeholders (eg, older people and their care partners, service providers and commissioners, education providers, professional bodies and advocacy organisations), to ensure the recommendations we develop are relevant and actionable.

\section{ETHICAL ISSUES}

Ethical approval will not be required to undertake this review. The interviews to be conducted as part of phase 3 will be undertaken with service staff, and therefore will require ethical approval by the study's sponsor (Bangor University).

\section{PROJECT OUTPUTS}

A number of products will be produced and processes engaged in as part of end-of-grant dissemination activity, including the following:

- A final and full research report, illustrated with vignettes of different practical examples and/or case studies to make findings relevant to the support workforce, NHS and social care managers, and a new framework for skills development for the support workforce for older people;

- An executive summary of the final report, suitable for use as a separate report for briefing NHS managers;

- A lay summary of the final report, suitable for use as a separate report for briefing the public;

- Benchmarking or quality assurance framework for interventions;

- Two open access publications: (1) a review protocol and (2) a findings paper that sets out an implementation plan of workforce development interventions training for the support workforce.

The project website will provide a real-time report of progress http://opswise.bangor.ac.uk/

Specifically, the study's outputs will provide: 
1. A clear description of the interventions that have been used and evaluated for improving the skills and care standards in the support workforce. This will include how they work in practice and their intended and unintended outcomes to enable NHS decision makers and policy makers to have an understanding of the range of strategies available, and the core assumptions about how they are supposed to work.

2. An explanation of the contextual influences underlying the challenges of designing and implementing support care workforce development interventions. Understanding context is not a central feature of traditional reviews, but for realist inquiry it is central. The impact of programmes and interventions are contingent on the conditions in which they are implemented, therefore a detailed explanation of this will provide service managers and policy makers with the information they need to address these issues locally.

3. An evidence informed framework of what works for whom and in what context in relation to interventions for improving skills and care standards in the assistant care workforce for older people. This could be used by managers and organisations to reform and enhance the support worker function by helping identify appropriate development interventions for different roles and to implement and evaluate new models of learning and development. For example, findings about effective interventions could be used to develop clear career development paths, and for improving the supervision and/or support offered to the workforce. This framework will be linked to personal development and career development frameworks, including the NHS Knowledge and Skills Framework, in order to promote implementation and maximise utility. In particular, we will suggest tailored mechanisms and interventions suitable for developing support workers, which can be used to strengthen these frameworks, and which may be of relevance across public services.

\section{DISCUSSION}

Syntheses of evidence about the effectiveness of workforce development interventions to enhance the knowledge and skills of the health and social care support workforce are urgently needed to meet the high-profile challenges to care standards for older people. Therefore, this review is timely and should provide important evidence of what works for support worker development interventions and programmes, to enhance understanding and provide clarity for older people's services.

The review findings have the potential to impact on policy and strategy and should provide guidance on how a workforce could be prepared for delivering care that is consistent and person-centred. ${ }^{19}$ Our findings have the potential to improve care provision for older people by theorising and synthesising evidence about the development of the support workforce which recognises the variety of ways and circumstances in which the holistic health and social care needs of older people are met. However, we are also cognisant that the review may uncover findings which affirm a growing concern that the boundaries between registered and support staff are becoming increasingly blurred. ${ }^{19}$ It is crucial that the evidence generated by the review connects and provides clarity across health and social care services, so that appropriate interventions of relevance to older people (and where appropriate their families/carers) can be implemented and sustained through education and development. Specifically, we will provide information about what workforce development programmes and interventions may work better in particular contexts and why. This can be expected to include the development, education and support offered to support workers and their supervisors ${ }^{45}$ and should also reflect the physical and emotional demands of providing care for people with complex and debilitating conditions. ${ }^{13}$

The transferability of research outputs will be enhanced through developing theoretically informed statements about 'what works' in workforce development, which are grounded in the reality of service delivery. Therefore, the findings from this review will relate to workforce interventions for support workers across different service settings, and therefore will likely be of interest beyond health and social care services.

Acknowledgements The authors would like to acknowledge Roger Williams, Stephen Edwards and Denise Fisher for their input into the development of the programme theory, and Dr Mark Smith for support with drafting the funded protocol.

Contributors All authors provided substantial contributions to protocol conception and design, drafting the article and revising it critically for intellectual content, and final approval of the version to be published.

Funding This work is supported by the National Institute Health Research (NIHR) Grant no. 12/129/32. This project is commissioned by the NIHR Health Services and Delivery Research Programme.

Competing interests Funding for this review was secured by JR-M and CB as joint chief investigators.

Ethics approval Bangor University, Bangor, UK.

Provenance and peer review Not commissioned; externally peer reviewed.

Open Access This is an Open Access article distributed in accordance with the Creative Commons Attribution Non Commercial (CC BY-NC 3.0) license, which permits others to distribute, remix, adapt, build upon this work noncommercially, and license their derivative works on different terms, provided the original work is properly cited and the use is non-commercial. See: http:// creativecommons.org/licenses/by-nc/3.0/

\section{REFERENCES}

1. Wise J. Number of 'oldest old' has doubled in the past 25 years. BMJ 2010;340:c3057.

2. Institute of Fiscal Studies. NHS and Social Care Funding: the outlook to 2021/22. 2012. http://www.nuffieldtrust.org.uk

3. Shield F, Enderby P, Nancarrow S. Stakeholder views of the training needs of an interprofessional practitioner who works with older people. Nurse Educ Today 2006;26:367-76.

4. Care Quality Commission. Dignity and Nutrition Inspection Programme: National Overview. 2011. http://www.cqc.org.uk

5. Parliamentary \& Health Service Ombudsman. Care and compassion? Report of the Health Service Ombudsman on ten investigations into 
NHS care of older people. London: The Stationary Office, 2011. http:// www.ombudsman.org.uk/care-and-compassion/home

6. Tadd W, Hillman A, Calnan S, et al. Dignity in practice: an exploration of the care of older adults in acute NHS Trusts. NIHR Service Delivery and Organisation Programme, 2011.

7. Commission for Dignity in Care. Delivering dignity: securing dignity in care for older people in hospitals and care homes. Joint Report from Independent Commission on Dignity, 2012. http://www. nhsconfed.org/Publications/reports/Pages/Delivering-Dignity.aspx

8. Rudd AG, Hoffman A, Down C, et al. Access to stroke care in England, Wales and Northern Ireland: the effect of age, gender and weekend admission. Age Aging 2007;36:247-55.

9. Gott M, Small N, Barnes S, et al. Older people's views of a good death in heart failure: implications for palliative care provision. Soc Sci Med 2008;67:1113-21.

10. Saks M, Allsop J, Chevannes M, et al. Review of health support workers. Report to the UK Departments of Health. Leicester: Faculty of Health and Community Studies, De Montfort University, 2000.

11. Spilsbury K, Adamson J, Atkin K, et al. Evaluation of The development and impact of assistant practitioners supporting the work of ward-based registered nurses in acute NHS (Hospital) Trusts in England. Final report. NIHR Service Delivery and Organisation Programme, 2010.

12. Nancarrow SA, Shuttleworth $P$, Tongue $A$, et al. Support workers in intermediate care. Health Soc Care Community 2005;13:338-44.

13. Schneider J, Scales K, Bailey S, et al. Challenging care: the role and experience of health care assistants in dementia wards. Final report. NIHR Service Delivery and Organisation Programme, 2010.

14. NHS Education for Scotland. Healthcare support workers. The development of the clinical support worker role: a review of the evidence. NHS Education for Scotland, 2010.

15. Skills for Care. Capable, competent, skilled. A workforce development strategy. Leeds: Skills for Care, 2011.

16. Janes N, Sidani S, Cott C, et al. Figuring it out in the moment: a theory of unregulated care providers' knowledge utilization in dementia care settings. Worldviews Evid Based Nurs 2008;5:13-24.

17. Manthorpe J, Martineau S, Moriarty J, et al. Support workers in social care in England: a scoping study. Health Soc Care Community 2010;18:316-24.

18. Griffiths $P$, Robinson S. Moving forward with healthcare support workforce regulation. London: National Nursing Research Unit, Kings College, 2010

19. Cavendish C. The Cavendish review: an independent review into healthcare assistants and support workers in the NHS and social care settings. 2013. https://www.gov.uk/government/publications/ review-of-healthcare-assistants-and-support-workers-in-nhs-andsocial-care

20. Kessler I, Heron P, Dopson S, et al. The nature and consequences of support workers in a hospital setting. Final report. NIHR Service Delivery and Organisation Programme, 2010.

21. Moran A, Enderby P, Nancarrow S. Defining and identifying common elements of and contextual influences on the roles of support workers in health and social care: a thematic analysis of the literature. J Eval Clin Pract 2010;17:1191-9.

22. Wakefield A, Spilsbury K, Atkin K, et al. Assistant or substitute: exploring the fit between national policy vision and local practice realities of assistant practitioner job descriptions. Health Policy 2009;90:286-95.

23. Nancarrow SA, Enderby $\mathrm{P}$, Moran AM, et al. The relationship between workforce flexibility and the costs and outcomes of older peoples' services. Final report. NIHR Service Delivery and Organisation Programme, 2010.

24. Bond J, Bamford C, Arksey $\mathrm{H}$, et al. Person- and carer-centred respite care for people with dementia: developing methods of evaluating the effectiveness of different models. Final report. NIHR Service Delivery and Organisation Programme, 2009.

25. Dreyfus SE, Dreyfus HL. A five stage model of the mental activities involved in directed skill acquisition. Unpublished Report supported by the Air Force Office of Scientific Research. Berkley: University of California, 1980.

26. Wong G, Greenhalgh T, Westhorp G, et al. RAMESES publication standards: realist synthesis. BMC Med 2013;11:21. http://www. biomedcentral.com/content/pdf/1741-7015-11-21.pdf

27. Wand T, White K, Patching J. Applying a realist(ic) framework to the evaluation of a new model of emergency department based mental health nursing practice. Nurs Inq 2010;17:231-9.

28. Hewitt $G$, Sims S, Harris R. The realist approach to evaluation research: an introduction. Int J Ther Rehabil 2012;19:250-9.

29. Gough D. Meta-narrative and realist reviews: guidance, rules, publication standards and quality appraisal. BMC Med 2013;11:22. http://www.biomedcentral.com/1741-7015/11/22

30. Pawson R. Evidence-based policy: a realist perspective. London: Sage Publications, 2006.

31. Rycroft-Malone J, McCormack B, Hutchinson A, et al. Realist synthesis: illustrating the method for implementation research. Implement Sci 2012;7:33.

32. Greenhalgh T, Wong G, Westhorp G, et al. Protocol-realist and meta-narrative evidence synthesis: evolving standards (RAMESES). BMC Med Res Methodol 2011;11:115.

33. McCormack B, Rycroft-Malone J, DeCorby K, et al. A realist review of interventions and strategies to promote evidence-informed healthcare: a focus on change agency. Implement Sci 2013;8:107. http://www.implementationscience.com/content/8/1/107

34. Dreyfus H, Dreyfus S. Mind over machine: the power of human intuition and expertise in the era of the computer. New York: Free Press, 1985

35. Bloom B. The taxonomy of educational objectives: the classification of educational goals. Handbook I, Cognitive Domain. London: Longman, 1956.

36. Kolb DA. Experiential learning. Experience as the source of learning and development. EnglewoodCliffs: Prentice Hall, 1984

37. Michie S, Fixsen D, Grimshaw JM, et al. Specifying and reporting complex behaviour change interventions: the need for a scientific method. Implement Sci 2009;4:40.

38. Prochaska JO, DiClemente CC. Transtheoretical therapy: towards a more integrative model of change. Psychother Theory Res Pract 1982;19:276-88.

39. McCormack B, Wright J, Dewer B, et al. A realist synthesis of evidence relating to practice development: findings from the literature review. Pract Dev Health Care 2007;6:25-55.

40. Rycroft-Malone J, Kitson A, Harvey G, et al. Ingredients for change: revisiting a conceptual framework. Qual Saf Health Care 2002;11:174-80.

41. Nutley S, Walters I, Davies HTO. Using evidence. How research can inform public services. Bristol: Policy Press, 2007

42. Easterby-Smith M. Disciplines of organizational learning: contributions and critiques. Hum Relations 1997;50:1085-113.

43. Raelin JA. Work-based learning in practice. J Workplace Learn 1997;10:280-3.

44. Dewing J. Chapter 15. Becoming and being active learners and creating active learning workplaces: the value of active learning in international practice development. In: McCormack B, Manley K, Wilson V, eds. Nursing and healthcare. Oxford: Blackwells, 2008:273-94.

45. Keeney S, Hasson F, McKenna H. Health care assistants: the views of managers of health care agencies on training and employment. J Nurs Manag 2005;13:83-92. 